\title{
Updates on the QCD phase diagram from lattice
}

\author{
Sayantan Sharma
}

\begin{abstract}
Different aspects of the phase diagram of strongly interacting matter described by quantum chromodynamics (QCD), which have emerged from the recent studies using lattice gauge theory techniques, are discussed. A special emphasis is given on understanding the role of the anomalous axial U(1) symmetry in determining the order of the finite temperature chiral phase transition in QCD with two massless quark flavors and tracing its origin to the topological properties of the QCD vacuum.
\end{abstract}

\section{Introduction}

Understanding the phase diagram of strongly interacting hadronic matter described by quantum chromodynamics (QCD) is of fundamental importance, as it enables us to explain the origin of mass of $99.9 \%$ of the visible matter in the present universe. It has motivated large scale experiments from the Large Hadron Collider at CERN to the Relativistic Heavy Ion Collider at Brookhaven. Unraveling the nature and phases of QCD is one of the most challenging problems in modern theoretical physics, since much of it is driven by non-perturbative interactions. Lattice QCD is one of the most successful non-perturbative methods available to us. Using lattice techniques, it has been conclusively demonstrated that the phase transition in 2+1-flavor QCD, at vanishingly small baryon densities that may have occurred in the early epoch of cosmological evolution of the universe, is a smooth crossover [1-3]. Bulk thermodynamic quantities like entropy density, pressure and the equation of state (EoS) at vanishingly small baryon densities are now known to a very high precision $[4,5]$ and more recently the continuum estimates for the EoS available at baryon densities as large as $\mu_{B} / T \sim 2.5$ [6]. Lattice techniques are now becoming sophisticated enough to provide fundamental insights on the more microscopic details of different phases of QCD, in particular, on the origins of chiral symmetry

The Institute of Mathematical Sciences, HBNI, Chennai 600113 India breaking and confinement that drive the phase transition in QCD. Understanding the mechanism behind the confinement of color degrees of freedom in gauge theories is one the most intriguing problems of contemporary research and any progress made towards understanding this phenomenon will definitely be an important milestone.

In this review article, updates and latest results on the QCD phase diagram at vanishingly small baryon densities, from very recent lattice studies will be discussed. Different phases of QCD as a function of the quark masses have been summarized in the famous Columbia plot. I will discuss about the current status of the Columbia plot and how a detailed understanding of it can in turn enrich our understanding of the phase diagram of QCD with physical quark masses. Moreover, I will further explain how the different phases in the Columbia plot provide us with insights about the intimate connection between chiral symmetry breaking and confinement. Both these phenomena are believed driven by topological properties of QCD, thus providing us with further insights about the vacuum structure of QCD. In recent years, the Columbia plot is studied on the lattice including a new axis to it, defined by an imaginary quark chemical potential. I will conclude this article with the new results coming from these studies. Throughout this article, the connection of the phase transition in QCD to the topological properties of the vacuum will be stressed. 


\section{The status of the Columbia plot of QCD at $\mu_{B}=0$}

The up and down quark masses in QCD are much lighter than its intrinsic scale, i.e $m_{l}=m_{u, d}<<\Lambda_{Q C D}$; hence, the $U_{L}(2) \times U_{R}(2)$ symmetry of the QCD action is very mildly broken. $U_{L}(2) \times U_{R}(2)$ is isomorphic to $S U(2)_{V} \times$ $S U(2)_{A} \times U_{B}(1) \times U_{A}(1)$ and QCD with two light and one heavier strange quark flavors, has to a very good approximation, a $S U(2)_{V} \times S U(2)_{A} \times U_{B}(1)$ symmetry. This symmetry is broken to $S U(2)_{V} \times U_{B}(1)$ as the temperatures are lowered below $\sim \Lambda_{Q C D}$, leading to chiral symmetry breaking. The anomalous $U_{A}(1)$ part is always broken due to quantum effects. Though the chiral symmetry is exact in the limit $m_{u}, m_{d} \rightarrow 0$, however, remnants of it exist in chiral observables. Thus even for QCD with physical quark masses, the temperature at which an inflection point exist for the subtracted chiral condensate is consistent with the one at which the chiral susceptibility or its disconnected part shows a maxima. An unweighted average of these temperature estimates in the continuum limit has allowed for a very precise determination of the pseudo-critical temperature $T_{c}=156.5 \pm 1.5 \mathrm{MeV}$ [7], to within a $1 \%$ precision.
The $U_{A}(1)$ part of the chiral/flavor symmetry is an anomalous symmetry; thus, there is no corresponding order parameter. From renormalization group studies of model quantum field theories with the same flavor symmetries as QCD, it has been observed that the order of phase transition for two flavor QCD depends on the effective magnitude of the $U_{A}(1)$ breaking at $T_{c}$ [8]. Further studies using the epsilon expansion and renormalization group [9] as well as the conformal bootstrap techniques [10] have revealed a possibility of a first-order or even a second-order phase transition of $U_{L}(2) \times U_{R}(2) / U_{V}(2)$ universality if the $U_{A}(1)$ is effectively restored near $T_{c}$. This is in contrast to an $O(4)$ second-order transition, if it remains broken. Magnitude of the effective breaking of $U_{A}(1)$, can only be determined non-perturbatively. Lattice techniques can and have contributed towards a more systematic understanding on this issue.

Before elaborating on it further, I would like to explain its consequences for the QCD phase diagram in the context of the Columbia plot. The current status of the Columbia plot is shown in Fig. 1, taken from Ref. [11]. The plot summarizes the fate of chiral phase transitions when

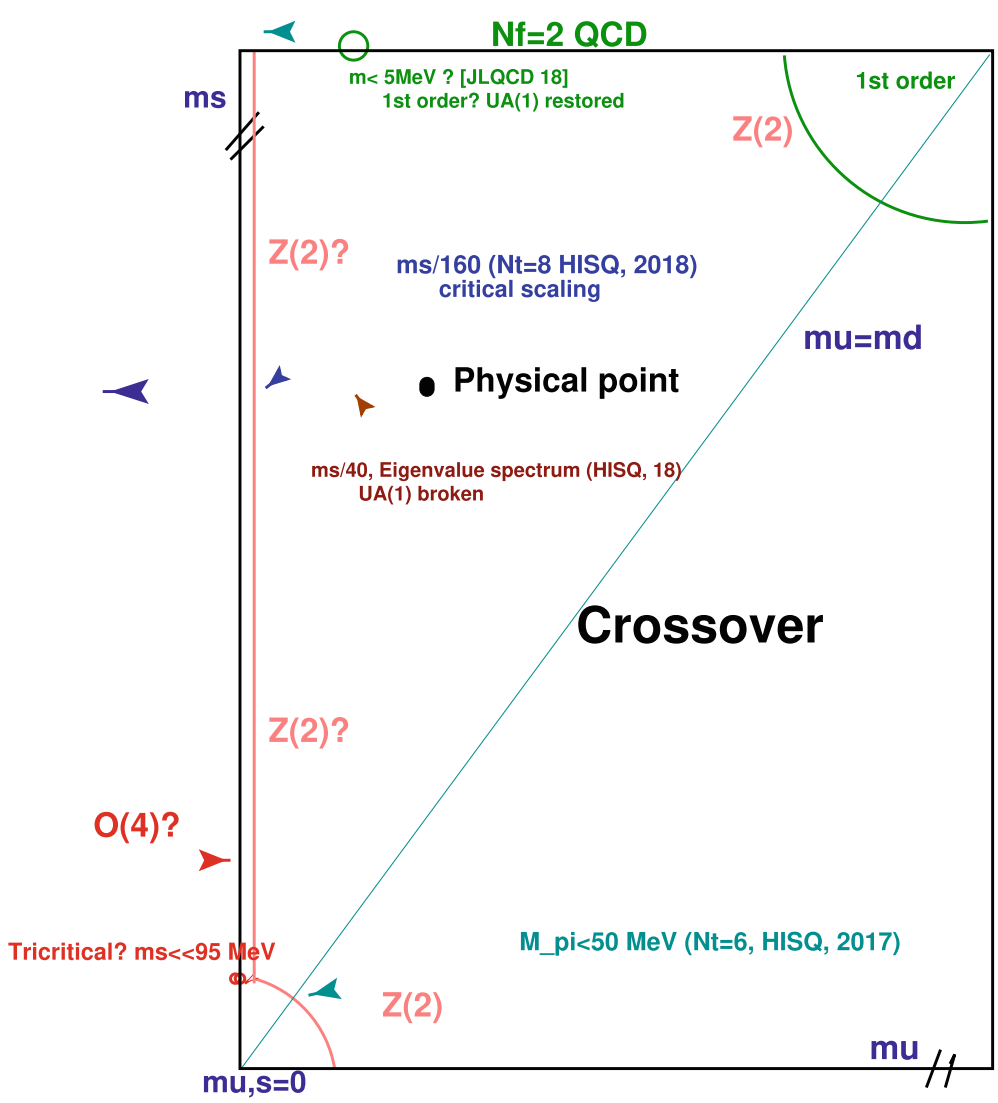

Fig. 1 The current status of Columbia plot from various lattice studies, most recent of which are shown as arrows on the diagram, taken from reference [11] 
the masses of the light and strange quark flavors are varied. QCD with physical quark masses lie in the crossover region which is extended over a range of $m_{u}, m_{s}$. The upper right corner of the plot is now well understood since for quark masses infinitely large, it corresponds to a $S U$ (3) gauge theory which has a first order transition [12]. This first-order region is separated from the crossover region by a $Z(2)$ second-order line. The lower left corner of the plot is yet to be understood comprehensively. From model quantum field theories with same symmetries as $N_{f}=3$ QCD, it is expected that a first-order region exist which should again be separated from the crossover region by a second-order $Z(2)$ line. Scaling studies of chiral susceptibilities along the very left part of the diagonal (which represents $m_{s}=m_{u, d}$ ) on $N_{\tau}=6$ lattices with a particular fermion discretization called the highly improved staggered quarks (HISQ), constrain the $Z(2)$ line to exist for pion masses $m_{\pi}<50 \mathrm{MeV}$ [13]. With yet another fermion discretization on the lattice known as clover improved Wilson fermions, the corresponding critical pion mass has been measured to be $m_{\pi}<170 \mathrm{MeV}$. This study at present is performed on rather coarse lattices [14]. However, it is important to note that irrespective of the choice of the fermion discretization on the lattice, the firstorder region tends to shrink when the lattice spacings are made finer [15], i.e., when one approaches the continuum limit. This picture seems to be realized also for $N_{f}=4$ QCD [16].

The second important issue that is still remains unresolved is whether this first-order region in the lower left corner of the plot ends in a tri-critical point in the chiral limit, i.e., $m_{u, d}=0$ and a finite $m_{s}$ or continues all the way to the $m_{s} \rightarrow \infty$ axis. Which of these two scenarios are realized in the continuum is ultimately related to the effective magnitude of the anomalous $U_{A}(1)$ symmetry. If $U_{A}(1)$ is effectively restored, the first-order region in the lower left hand corner of the plot may extend as a tiny strip parallel to the $m_{s}$ axis all the way to $m_{s} \rightarrow \infty$, separated from the crossover region by the $Z(2)$ line. On this $Z$ (2) line, the values of $m_{u, d}$ is much smaller than physical quark mass. To introduce the readers to the latest developments, arrows are marked on the Columbia plot in Fig. 1, to indicate the directions which are pursued by the most recent lattice studies in this regard. In summary,

- The green arrow shows the approach for $N_{f}=2$ QCD, followed by one of the recent lattice studies $[17,18]$. With the current lattice volume $(2.4 \mathrm{fm})^{3}$ and inverse lattice spacing $a^{-1}=2.6 \mathrm{GeV}$, the results seem to suggest that $U_{A}(1)$ is restored at $\sim 1.1 T_{c}$ for $m_{u, d} \lesssim 5 \mathrm{MeV}$. On the other hand, expectations from $N_{f}=3$ QCD suggest that in the continuum, the first-order region, if it survives and continues from the lower left corner to the $N_{f}=2$ axis, will very narrow, characterized by $m_{u, d}<<5 \mathrm{MeV}$. It is thus a challenge to reconcile both these results; perhaps, it will be resolved in the continuum limit.

- The blue line on the Columbia plot shows the other approach that was pursued by an independent lattice study [19], where the strange quark mass $m_{s}$ is fixed to its physical value and $m_{u, d}$ is successively reduced to check whether one approaches the $Z(2)$ line or an $O(4)$ second-order line. New results on chiral susceptibility for $N_{\tau}=8,12$ lattices with HISQ fermions suggest that its peak height decreases with increasing lattice volumes, ruling out first-order phase transition for $M_{\pi}>80 \mathrm{MeV}$. Scaling studies of the chiral condensate seems to rule out $Z(2)$ scaling for $M_{\pi}>55 \mathrm{MeV}$, implying that the $Z(2)$ line if it exists would be further left towards the $m_{u, d}=0$ axis of the Columbia plot.

- Yet, another approach pursued was to measure the eigenvalue distribution of the QCD Dirac operator and understand the fate of $U_{A}(1)$ from the characteristics of the eigenvalue spectrum. A recent lattice study has also followed along the path marked by the blue arrow on the Columbia plot, to verify if $U_{A}(1)$ remains broken as light quark mass is successively reduced from its physical value [20]. If indeed $U_{A}(1)$ remains strongly broken, one will directly encounter the $O(4)$ line instead of a $Z(2)$ second-order line when moving towards the chiral limit $m_{u, d} \rightarrow 0$. The eigenvalue densities as observed different pion masses $M_{\pi} \sim 160,140,110 \mathrm{MeV}$ seem to support the existence of the $O(4)$ line [20].

- A recent study was performed by considering $N_{f}$ as a continuous parameter and study the fate of the chiral phase transition as a function of $N_{f}$ [21]. Starting with the first-order region corresponding to $N_{f}=3$ QCD with finite quark masses, one can zoom in to the tri-critical scaling regime to constrain the minimum number of light quark flavors for which the tri-critical line exist, $N_{f}^{\text {tric }}$. Using the scaling relation $m_{q} \sim\left(N_{f}-N_{f}^{\text {tric }}\right)^{5 / 2}$, this present study on $N_{\tau}=4$ lattices concludes that the $N_{f}^{\text {tric }}<2$, which seems to suggest a first-order transition for two flavor QCD. These results are being further verified in the continuum limit.

Emphasizing again that the $U_{A}(1)$ is not the symmetry of the QCD partition function, thus, there is no corresponding order parameter. However, one can indirectly quantify its effective magnitude from different observables. One of the earliest suggestions was to look for the degeneracy of the integrated two-point correlation functions of iso-triplet pseudo-scalar and scalar mesons [22] in the chiral symmetry restored phase. In two flavor QCD, the possible meson correlation functions are: 


$$
\begin{aligned}
& \chi_{\sigma}=\frac{1}{2} \int d^{4} x\langle\sigma(x) \sigma(0)\rangle, \chi_{\delta}=\frac{1}{2} \int d^{4} x\left\langle\delta^{i}(x) \delta^{i}(0)\right\rangle \\
& \chi_{\eta}=\frac{1}{2} \int d^{4} x\langle\eta(x) \eta(0)\rangle, \chi_{\pi}=\frac{1}{2} \int d^{4} x\left\langle\pi^{i}(x) \pi^{i}(0)\right\rangle .
\end{aligned}
$$

Here, the meson operators are defined as $\sigma(x)=\bar{\psi}_{l}(x) \psi_{l}(x), \delta^{i}(x)=\bar{\psi}_{l}(x) \tau^{i} \psi_{l}(x), \eta(x)=$ $i \bar{\psi}_{l}(x) \gamma^{5} \psi_{l}(x), \pi^{i}(x)=i \bar{\psi}_{l}(x) \tau^{i} \gamma^{5} \psi_{l}(x), \tau$ being the isospin operator and $\psi_{l}$ denotes the field operator corresponding to the two degenerate light quark flavors. When the chiral symmetry is restored then $\chi_{\pi}=\chi_{\sigma}$ and similarly $\chi_{\eta}=\chi_{\delta}$. If one further demands anomalous $U_{A}(1)$ restoration, then it would result in $\chi_{\pi}=\chi_{\eta}$ which due to chiral symmetry restoration would result in $\chi_{\pi}=\chi_{\delta}$. Thus the difference between the integrated correlation functions $\chi_{\pi}-\chi_{\delta}$ is sensitive to the $U_{A}(1)$.

The integrated correlators can in turn be written in terms of the eigenvalues $\lambda$ and density $\rho(\lambda)$ of the QCD Dirac operator since $\chi_{\pi}-\chi_{\delta}=\int d \lambda \frac{4 m_{l}^{2} \rho(\lambda)}{\left(\lambda^{2}+m_{l}^{2}\right)^{2}}$. Therefore, typical characteristics of the eigenvalue spectrum as a function of temperature can provide us with information about the fate of the $U_{A}(1)$ [23]. One way to trivially realize $U_{A}(1)$ restoration along with the chiral symmetry is to have a gap in the infrared region of the eigenvalue spectrum, i.e., $\rho(\lambda \rightarrow 0)=0$ [24]. A recent theoretical study has suggested that is important to look at even higher order correlation functions in all these mesonic quantum number channels [25]. In the chiral limit of two-flavor QCD, $U_{A}(1)$ breaking effects can be invisible in up to 6-point correlation functions in the scalarpseudo-scalar channel if the eigenvalue density goes as $\rho(\lambda) \sim \lambda^{3}[25]$.

Recent measurements of $\chi_{\pi}-\chi_{\delta}$ in two flavor QCD with fermions with nearly exact chiral symmetry on the lattice (along the direction of the green arrow in the Columbia plot) report that $U_{A}(1)$ may be restored at about $1.1 T_{c}$ [18]. Current systematics are still large to make any predictions closer to $T_{c}$. For physical quark masses, the $U_{A}(1)$ breaking is still finite on lattices of size $48^{3} \times 12$ which tend to decrease to zero in the limit $m_{l} \rightarrow 0[17,18]$. On the other hand, pursuing along the blue arrow on the Columbia plot, the eigenvalue density of the Dirac operator in $2+1$ flavor QCD (by fixing the strange quark mass $m_{s}$ to its physical value and successively reducing the light quark masses towards the chiral limit) does not show any trends towards opening up of a gap in the infrared region [20]. In fact, the infrared part of the spectrum has a similar behavior $\rho(\lambda) \sim \lambda$ at around $1.1 T_{c}$, when the light quark masses are reduced from $m_{l}=m_{s} / 20$ [26] to $m_{s} / 80$. Even though for the given lattice sizes studied, the QCD Dirac operator has millions of eigenvalues; the first $\sim 100$ of them contribute maximally to the $U_{A}(1)$ breaking observable, described earlier. An appropriately renormalized observable $m_{s}^{2}\left(\chi_{\pi}-\chi_{\delta}\right) / T^{4}$, reported in the same work, remains finite even for light quark mass $m_{l}=$ $m_{s} / 40$ at temperatures up to $1.1 T_{c}$. This suggests $U_{A}(1)$ breaking may as well survive towards the chiral limit [20].

An observable that measures the localized topological fluctuations of QCD vacuum at any temperature $T$ is the topological susceptibility defined as $\chi_{t}=\frac{T}{V}\left\langle Q^{2}\right\rangle$. Here, $Q$ is the topological charge of a typical gauge configuration and the angular bracket denotes quantum averaging and also over the Euclidean space-time volume. The topological susceptibility can be further related to the disconnected part of the meson correlators $\chi_{\eta}$ introduced earlier in Eq. 1,

$$
\chi_{t}=m_{l}^{2} \chi_{5, \text { disc }} .
$$

We recall here that the susceptibilities in the different quantum number channels are related as:

$$
\chi_{\sigma}=\chi_{\delta}+2 \chi_{\text {disc }}, \chi_{\eta}=\chi_{\pi}-2 \chi_{5, \text { disc }},
$$

where the $\pi$ and $\delta$-meson correlators only have connected parts. In the chiral symmetry restored phase of two-flavor QCD, Ward identities thus ensure that $\chi_{t}=$ $\frac{1}{2} m_{l}^{2}\left(\chi_{\pi}-\chi_{\delta}\right)$. This identity points to the fact that the axial $U(1)$ symmetry breaking or its restoration as a function of temperature is of topological origin. This identity has been verified to hold even for $2+1$ flavor QCD with nearly physical quark masses [27], showing that $\chi_{t}$ is an observable that can be used to determine the extent of effective breaking of the anomalous $U(1)$ symmetry in the chiral symmetry restored phase. The temperature dependence of the continuum extrapolated values for $\chi_{t}$ in QCD matches exactly with the expectations from a dilute instanton gas approximation (DIGA) for temperatures $T \gtrsim 3 T_{c}$ [27-30], whereas a non-trivial temperature dependence is observed for $T_{c}<T<3 T_{c}$ [27, 31]. New results for $\chi_{t}$ with a different fermion discretization in $2+1+1$ QCD have also confirmed this overall picture [32].

Just above $T_{c}$, the temperature dependence for $\chi_{t}$ is unambiguously different from the expectations of DIGA $[27,31]$, the origin of which is not yet understood. This reemphasizes the fact that the axial $U(1)$ symmetry is not effectively restored above $T_{c}$, and thus, the QCD vacuum does not immediately go over to a trivial dilute instanton gas regime. Can this give us some hints about the topological origin of confinement? We recall that at finite temperatures, the eigenvalues of Polyakov loop at spatial infinity (the holonomy values) characterize the instanton solution. For trivial holonomy, the finite action solution for the non-Abelian gauge fields at non-zero temperatures is known and termed as caloron [33]. Calorons with non-trivial holonomy $[34,35]$ in $S U(N)$ gauge theory consists of $N$ constituent instanton-dyons or simply dyons, which carry a fraction $1 / N$ of the net topological charge 
of the caloron. Moreover, dyons carry both color electric and magnetic charges. Whereas calorons with trivial holonomy cannot explain confinement in gauge theories, mean-field studies of dyon gas hints to the fact that they interact with the holonomy potential and thus driving towards confinement [36].

Earlier lattice studies [37, 38] have reported on the observation of dyons in pure gauge theory as well as in QCD respectively. On the lattice, the gauge ensembles are generated during a Monte-Carlo evolution with (anti)periodic boundary conditions along the temporal direction for (fermion) gauge fields respectively; thus, an isolated dyon which carries a non-trivial chromo-magnetic charge cannot exist. However, zero modes of a probe or valence Dirac operator, with generalized temporal periodicity phase $\psi(\tau+\beta)=\mathrm{e}^{i \phi} \psi(\tau)$, will detect the dyon whose action is characterized by the difference between those eigenvalues of the Polyakov loop within which the phase $\phi$ lies. Recently, this technique has been utilized to not only detect dyons but also identify the different species of dyons in the temperature range $T_{c}<T<1.2 T_{c}$ $[39,40]$. The density profiles of zero-mode wave functions of the valence QCD Dirac operator and their characteristic fall-off at large distances have been suggested as one of the observables to identify different species of dyons on the lattice. Moreover, the eigenvalue spectrum of the nearzero modes of the valence Dirac operator with different periodicity phases [40] show distinct features corresponding to the different species of dyons. To illustrate this point, we refer to Fig. 2 from [40]. The density of near-zero eigenvalues corresponding to the L-dyons (anti-periodic boundary condition or $\phi=\pi$ ) depletes at $T \sim 1.1 T_{c}$, signaling the restoration of chiral symmetry. However, the infrared part of the eigenvalue density corresponding to M-dyons $(\phi= \pm \pi / 3)$ is significantly larger than the Ldyons, which would imply presence of a large number of closely situated M-dyon pairs at high temperatures as compared to the L-dyons.

At higher temperatures $T>2 T_{c}$, the holonomy is trivial but there may be localized fluctuations of the Polyakov loop, which has been conjectured to provide the disordered landscape required to localize bulk eigenfunctions of the QCD Dirac operator [41]. Such localization of the Dirac eigenvalues have been reported in a recent lattice study in 2+1+1 flavor QCD [42].

\section{Adding a new dimension to the Columbia plot}

Till now, we have been discussing the conventional twodimensional Columbia plot. Imposing an additional third dimension in a form of an imaginary chemical potential [43] $i \mu_{q}$ can impose further constraints on the conventional Columbia plot. The QCD partition function in presence of $i \mu_{q}$ is free from the infamous sign-problem and has the property that $Z\left(\frac{\mu_{q}}{T}\right)=Z\left(-\frac{\mu_{q}}{T}\right)$ and $Z\left(\frac{\mu_{q}}{T}\right)=$ $Z\left(\frac{\mu_{q}}{T}+\frac{2 n i}{3} \pi\right)$ where $n \in \mathcal{Z}$. The center of the gauge group is a symmetry of the pure gauge theory partition function but remains broken when quark fields are introduced. However, in the presence of an imaginary quark chemical potential, the center symmetry is a good symmetry of QCD with finite quark masses again. The phase of the Polyakov loop is an observable in this case, which will identify the different $Z$ (3) sectors as one varies

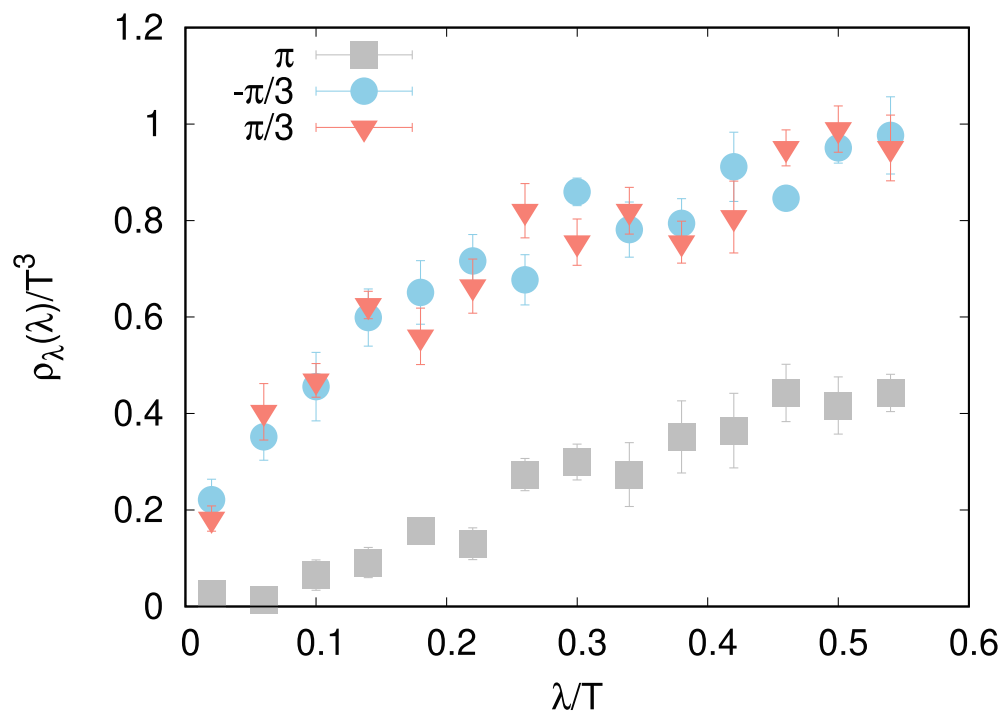

Fig. 2 The eigenvalue distribution of a valence Dirac operator (overlap operator) with temporal periodicity phases $\pi,-\pi / 3, \pi / 3$ respectively on $2+1$ flavor QCD gauge ensembles. It is evident from the plot that whereas the chiral condensate in QCD due to the L-dyons (corresponding to the usual anti-periodic boundary condition given by $\phi=\pi$ ) vanishes at around $1.1 T_{c}$, the contributions from $\mathrm{M}$-dyons survive. This figure has been taken from Ref. [40] 
$i \mu_{q}$. The Roberge-Weiss (RW) points characterized by $\mu_{q}=(2 n+1) \frac{i \pi T}{3}[44]$ represent the transition between adjacent center sectors, which is first order for high temperatures and a smooth crossover for lower temperatures. From the continuity of the free energy, the first-order lines should end in a second-order RW end-point.

How do the deconfinement and chiral transitions at $\mu_{q}=0$ connect to the RW points? From reflection symmetry of the partition function, the first-order lines for QCD with very heavy quark masses $m_{q}$ are expected to meet at the RW point, which will be a triple point. For intermediate values of $m_{q}$, the crossover curve at $\mu_{q}=0$ may meet at the RW end-point, expected to be in the $Z$ (2) universality class. In the chiral limit, the scenario is still far from being understood. Numerical simulations on relatively small $N_{\tau}=4$ lattices with staggered fermions have shown a first order RW transition for both $N_{f}=$ $2,3[43,45]$, which seems to survive in the chiral limit [46]. This scenario was later confirmed in studies with Wilson fermions [47]. The discussion in this section is succinctly summarized in the modified Columbia plot at $\mu_{q}=\mu_{B} / 3=i \pi T / 3$, shown in Fig. 3, taken from [11].

Now, I would like to elaborate on the consequences of these results obtained in the imaginary chemical potential plane to the nature of the $N_{f}=2$ chiral transition at $\mu_{q}=0$. If the $N_{f}=2$ chiral transition at $i \mu_{q}=0$ is second (first) order, then the first-order RW transition will end in a tri-critical point for $\mu_{q}^{2}<0\left(\mu_{q}^{2}>0\right)$ respectively. This opens up a new possibility to understand the nature of chiral phase transition and the role of $U_{A}(1)$ for $N_{f}=2$ QCD. Indeed, the first lattice study along this line [46], performed with staggered fermions on $N_{\tau}=4$ for different lattice volumes with $N_{s}=8,12,16$, reported a tri-critical point at $\mu_{q}^{2}=0.85(5) T^{2}$ which seemed to suggest that $N_{f}=2$ chiral transition at $\mu_{q}=0$ is first order at least on coarser lattices. Subsequently improved versions of staggered fermions have been used to reduce lattice cutoff effects. By fixing the $m_{s}$ to its physical value and reducing the $m_{l}=m_{u, d}$ at $\mu_{q}=i \pi T / 3$, i.e., along the blue line shown on the lower RW plane of Fig. 3 , the recent results [48] report that a first-order RW transition is not observed for $M_{\pi} \geq 50 \mathrm{MeV}$. In the continuum limit, it would imply that this first-order region, when it continues to the $\mu_{q}=0$ plane would be a very narrow strip parallel to the $m_{s}$ axis. Remarkably, the chiral and RW transition seem to follow each other as one reduces the $m_{u, d}$ [48].

Furthermore, if the end-point of the line of first-order RW transitions is of second order, then it would belong to the three-dimensional Ising universality class. The imaginary part of the Polyakov loop $L$ would behave like the

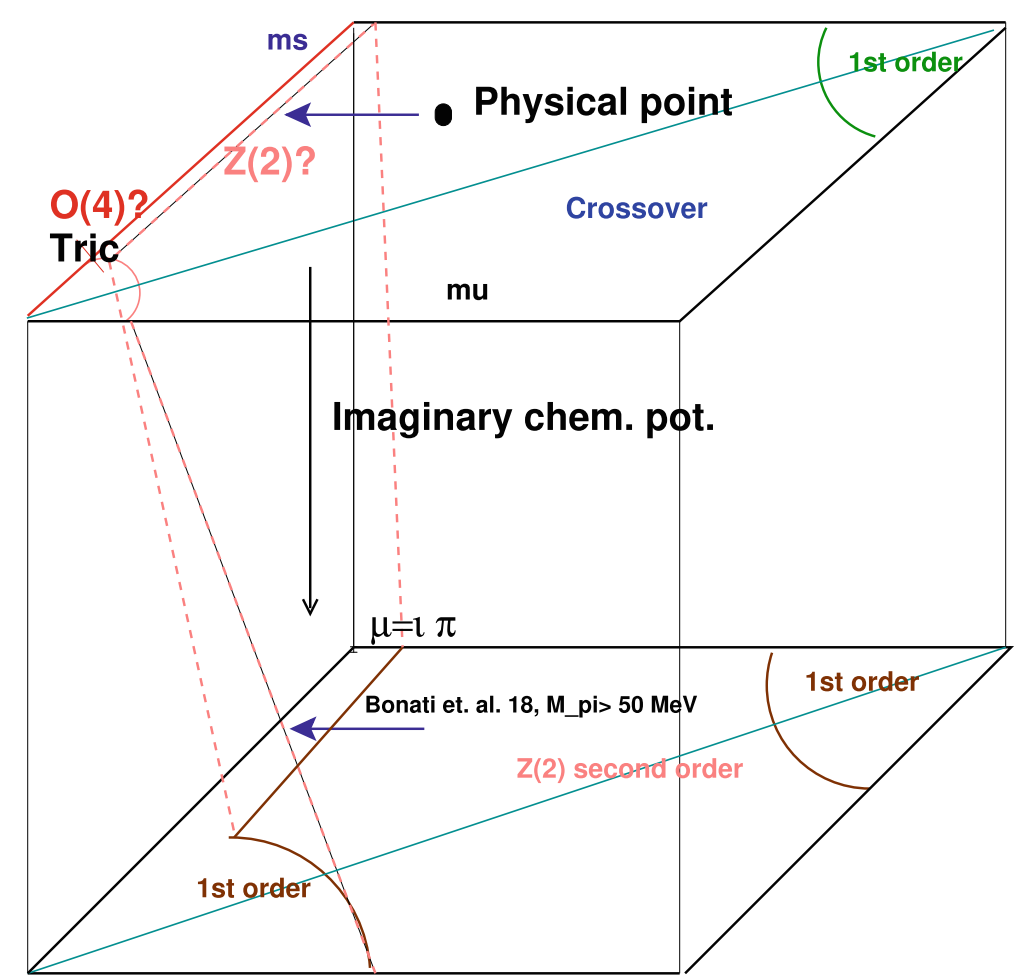

Fig. 3 Current status of the Columbia plot with an additional direction added to it which is characterized by the imaginary baryon chemical potential (which is a third of the quark chemical potential $\mu_{q}$ ) normalized by the temperature, $\mu_{B} / T$, taken from [1 1]. The lower plane is the current understanding we have of the generalized Columbia plot at $\mu_{B} / T=3 \mu_{q} / T=i \pi$ 


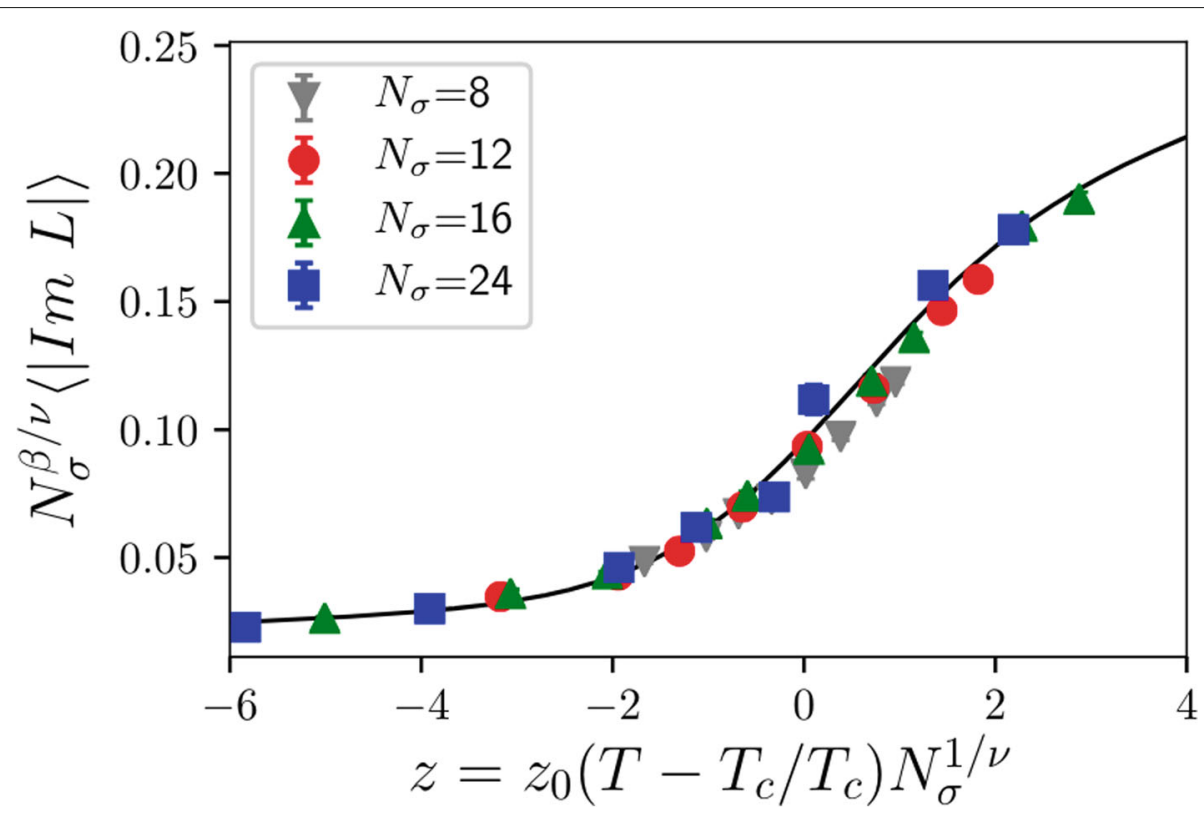

Fig. 4 The $Z$ (2) scaling of the expectation value of the imaginary part of Polyakov loop near the chiral crossover transition temperature $T_{c}$ for different lattice volumes, taken from [49]

magnetization order parameter of the Ising model. This is due to the fact that under $Z$ (2) transformation, the real part of Polyakov loop does not change sign whereas its imaginary part does. Scaling studies performed [49] with the expectation values of $\langle|\operatorname{Im} L|\rangle$, for different lattice volumes, $N_{\tau}=4$ and $N_{\sigma}=8-24$ around the chiral crossover transition temperature $T_{c} \sim 200 \mathrm{MeV}$ and $M_{\pi}=135-90$ $\mathrm{MeV}$ and on the $3 \mu_{q} / T=i \pi$ plane, confirm a secondorder $Z(2)$ scaling, essentially supporting the previous findings (Fig. 4) [48].

\section{Summary and outlook}

In this review article, the most recent findings about the QCD phase diagram arising from lattice studies is outlined in the context of the Columbia plot. Very new insights about the order of the chiral phase transition for $N_{f}=2$ QCD has been obtained both from the study of its eigenvalue spectrum (and integrated meson correlators) and indirectly from the scaling studies of imaginary part of the Polyakov loop expectation values at an imaginary baryon chemical potential $\mu_{B}=i \pi T$. Though most studies argue for a second-order $O(4)$ phase transition, however, no final conclusion has yet been achieved. The order of chiral phase transition at $N_{f}=2$ is intimately connected to the effective magnitude of the anomalous axial $U(1)$ symmetry breaking. It has been stressed in this article that the origin of this effective breaking of axial $U(1)$ is related to the topological fluctuations of the QCD vacuum hence to quantify it is one of the most challenging problems in lattice field theory. Ultimately, a comprehensive understanding of this phenomenon will in turn advance our knowledge about the mechanism of confinement and chiral transition in QCD with physical quark masses.

\section{Acknowledgements}

The author gratefully acknowledges support from the Department of Science and Technology, Govt. of India through a Ramanujan fellowship.

Authors' contributions

The author read and approved the final manuscript.

\section{Declarations}

\section{Competing interests}

The author declares that he has no competing interests.

Received: 16 November 2020 Accepted: 23 February 2021

Published online: 27 May 2021

\section{References}

1. Y. Aoki, G. Endrodi, Z. Fodor, S. D. Katz, K. K. Szabo. Nat. 443, 675 (2006). hep-lat/0611014

2. A. Bazavov, et al. Phys. Rev. D. 85, 054503 (2012). arXiv:1111.1710 hep-lat

3. T. Bhattacharya, et al. Phys. Rev. Lett. 113(8), 082001 (2014). arXiv:1402.5175 hep-lat

4. S. Borsanyi, et. al. Phys. Lett. B. 730, 99 (2014). arXiv:1309.5258 hep-lat

5. A. Bazavov, et al, HotQCD Collaboration. Phys. Rev. D. 90, 094503 (2014). arXiv:1407.6387 hep-lat

6. A. Bazavov, et al. Phys. Rev. D. 95(5), 054504 (2017). arXiv:1701.04325 hep-lat

7. A. Bazavov, et al, HotQCD Collaboration. Phys. Lett. B. 795, 15 (2019). arXiv:1812.08235 hep-lat

8. R. D. Pisarski, F. Wilczek. Phys. Rev. D. 29, 338 (1984)

9. A. Pelissetto, E. Vicari. Phys. Rev. D. 88(10), 18 (2013). arXiv:1309.5446 hep-lat

10. Y. Nakayama, T. Ohtsuki. Phys. Rev. D. 91 (2), 021901 (2015). arXiv:1407.6195 hep-th

11. S. Sharma. PoS. LATTICE2018, 009 (2019). arXiv:1901.07190 hep-lat 
12. G. Boyd, J. Engels, F. Karsch, E. Laermann, C. Legeland, M. Lutgemeier, B. Petersson. Nucl. Phys. B. 469, 419-444 (1996). arXiv:hep-lat/9602007 hep-lat

13. A. Bazavov, et. al. Phys. Rev. D. 95(7) (2017). arXiv:1701.03548 hep-lat

14. X. Y. Jin, et. al. Phys. Rev. D. 96(3) (2017). arXiv:1706.01178 hep-lat

15. P. de Forcrand, M. D'Elia. PoS LATTICE. 2016 (2017). arXiv:1702.00330 hep-lat

16. H. Ohno, et. al. PoS(LATTICE2018), 174. arXiv:1812.01318 hep-lat

17. A. Tomiya, et. al. Phys. Rev. D. 96(3), 034509 (2017). arXiv:1612.01908 hep-lat

18. S. Aoki, et. al. JLQCD. arXiv:2011.01499 hep-lat

19. H. T. Ding, P. Hegde, O. Kaczmarek, F. Karsch, A. Lahiri, S. T. Li, S. Mukherjee, H. Ohno, P. Petreczky, C. Schmidt, et al. Phys. Rev. Lett. 123(6), 062002 (2019). arXiv:1903.04801 hep-lat

20. L. Mazur, O. Kaczmarek, E. Laermann, S. Sharma. PoS. LATTICE2018, 153 (2019). arXiv:1811.08222 hep-lat

21. F. Cuteri, O. Philipsen, A. Sciarra. Phys. Rev. D. 97(11), 11 (2018). POS(LATTICE2018) 170

22. E. V. Shuryak. Comments Nucl. Part. Phys. 21(4), 235 (1994). hep-ph/9310253

23. S. H. Lee, T. Hatsuda. Phys. Rev. D. 54, 1871-1873 (1996). arXiv:hep-ph/9601373 hep-ph

24. A. Bazavov, et. al, HotQCD Collaboration. Phys. Rev. D. 86, 094503 (2012). arXiv:1205.3535 hep-lat

25. S. Aoki, H. Fukaya, Y. Taniguchi. Phys. Rev. D. 86, 12 (2012). arXiv:1209.2061 hep-lat

26. V. Dick, F. Karsch, E. Laermann, S. Mukherjee, S. Sharma. Phys. Rev. D. $91(9)$, 094504 (2015). arXiv:1502.06190 hep-lat

27. P. Petreczky, H. P. Schadler, S. Sharma. Phys. Lett. B. 762, 498 (2016). arXiv:1606.03145 hep-lat

28. J. Frison, R. Kitano, H. Matsufuru, S. Mori, N. Yamada. JHEP. 1609, 021 (2016). arXiv:1606.07175 hep-lat

29. S. Borsanyi, et. al. Nat. 539(7627), 69 (2016). arXiv:1606.07494 hep-lat

30. C. Bonati, M. D'Elia, G. Martinelli, F. Negro, F. Sanfilippo, A. Todaro. JHEP. 11, 170 (2018). arXiv:1807.07954 hep-lat

31. C. Bonati, et. al. JHEP. 1603, 155 (2016). arXiv:1512.06746 hep-lat

32. F. Burger, E. M. Ilgenfritz, M. P. Lombardo, A. Trunin. Phys. Rev. D. 98(9), 094501 (2018). arXiv:1805.06001 hep-lat

33. B. J. Harrington, H. K. Shepard. Phys. Rev. D. 17 (1978)

34. T. C. Kraan, P. van Baal. Phys. Lett. B. 435, 389 (1998). hep-th/9806034

35. K. M. Lee, C. h. Lu. Phys. Rev. D. 58, 025011 (1998). hep-th/9802108

36. D. Diakonov. Nucl. Phys. Proc. Suppl. 195, 5 (2009). arXiv:0906.2456 hep-ph

37. C. Gattringer. Phys. Rev. D. 67, 034507 (2003). hep-lat/0210001

38. V. G. Bornyakov, et. al. Phys. Rev. D. 93(7), 074508 (2016). arXiv:1512.03217 hep-lat

39. R. N. Larsen, S. Sharma, E. Shuryak. Phys. Lett. B. 794, 14-18 (2019). arXiv:1811.07914 hep-lat

40. R. N. Larsen, S. Sharma, E. Shuryak. Phys. Rev. D. 102(3), 034501 (2020). arXiv:1912.09141 hep-lat

41. F. Bruckmann, T. G. Kovacs, S. Schierenberg. Phys. Rev. D. 84, 034505 (2011). arXiv:1 105.5336 hep-lat

42. L. Holicki, E. M. Ilgenfritz, L. von Smekal. arXiv:1810.01130 hep-lat; POS(LATTICE2018), 180

43. P. de Forcrand, O. Philipsen. Phys. Rev. Lett. 105, 01 (2010). arXiv:1004.3144 hep-lat

44. A. Roberge, N. Weiss. Nucl. Phys. B. 275, 734 (1986)

45. M. D'Elia, F. Sanfilippo. Phys. Rev. D. 80, 01 (2009). arXiv:0909.0254 hep-lat

46. C. Bonati, P. Forcrand, M. D'Elia, O. Philipsen, F. Sanfilippo. Phys. Rev. D. 90(7), 074030 (2014). arXiv:1408.5086 hep-lat

47. C. Czaban, F. Cuteri, O. Philipsen, C. Pinke, A. Sciarra. Phys. Rev. D. 93(5), 054507 (2016). arXiv:1512.07180 hep-lat

48. C. Bonati, E. Calore, M. D'Elia, M. Mesiti, F. Negro, F. Sanfilippo, S. F. Schifano, G. Silvi, R. Tripiccione. Phys. Rev. D. 99(1), 014502 (2019). arXiv:1807.02106 hep-lat

49. J. Goswami, F. Karsch, A. Lahiri, C. Schmidt. PoS. LATTICE2018, 162 (2018). arXiv:1811.02494 hep-lat

\section{Publisher's Note}

Springer Nature remains neutral with regard to jurisdictional claims in published maps and institutional affiliations. 